\title{
ALGORITHMS
}

\section{Algorithms for managing the common trauma patient}

J John, MB ChB

Department of Urology, Frere Hospital, East London Hospital Complex, East London, South Africa

Corresponding author: J John (jeffveenajohn@gmail.com)

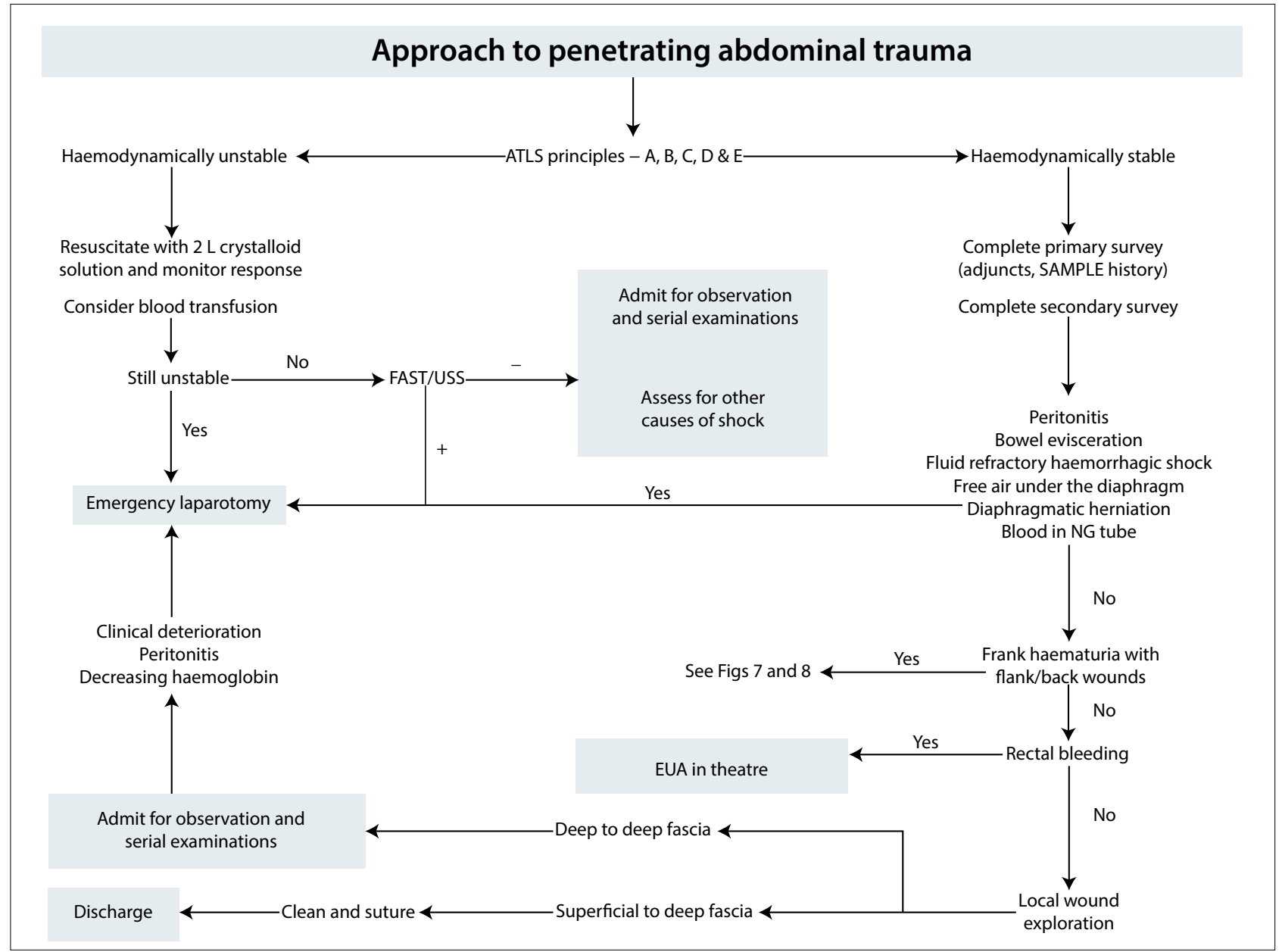

Fig. 1. Approach to penetrating abdominal trauma (ATLS = advanced trauma life support; FAST/USS $=$ focused assessment with sonography in traumal ultrasound scan; EUA = examination under anaesthesia; $C T=$ computed tomography; $N G=$ nasogastric; SAMPLE = signs and symptoms, allergies, medications, pertinent medical history, injuries, illnesses, last meal/intake, events leading up to the injury and/or illness). 


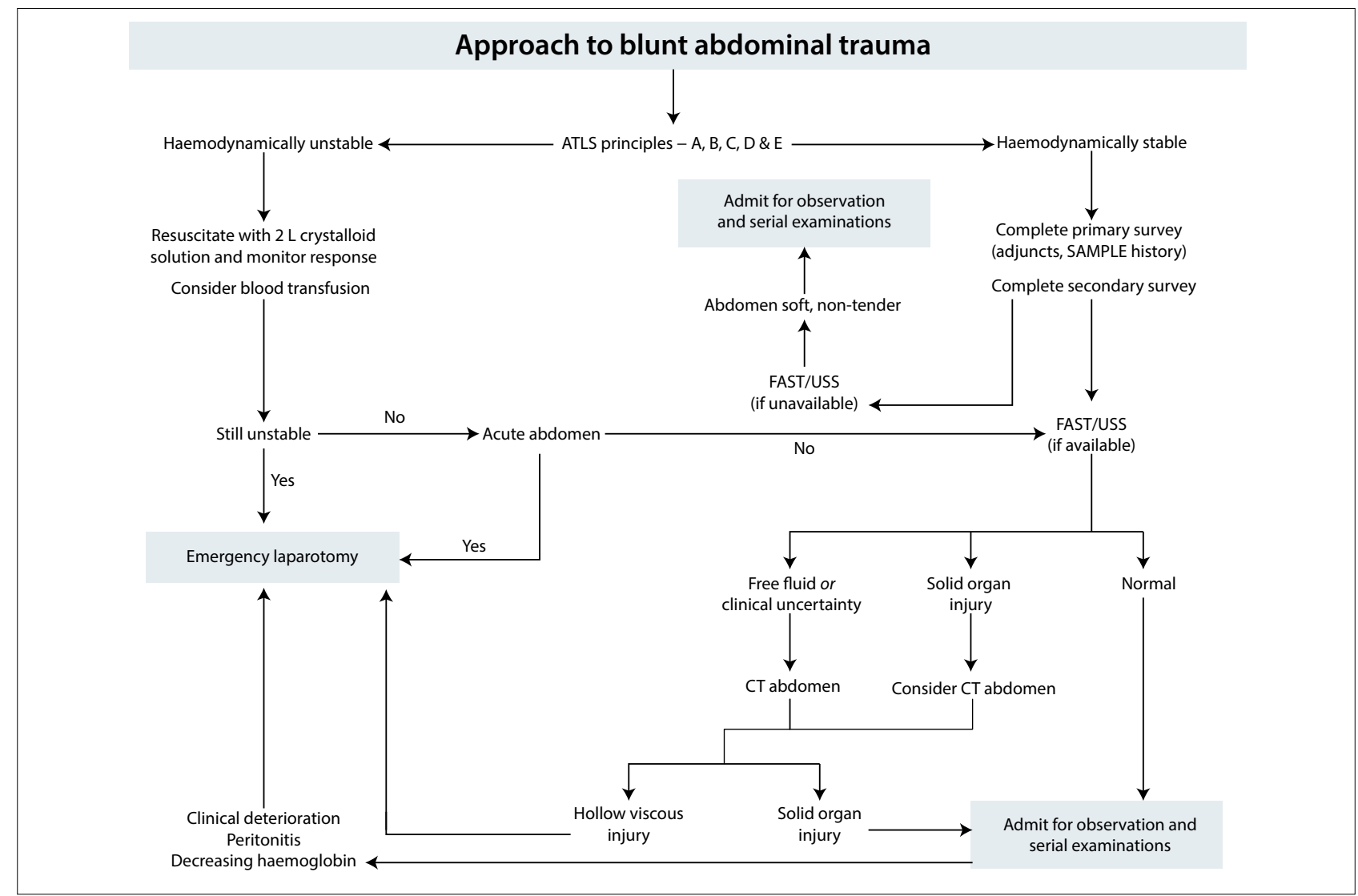

Fig. 2. Approach to blunt abdominal trauma (ATLS = advanced trauma life support; FAST/USS = focused assessment with sonography in trauma/ultrasound scan; CT = computed tomography; SAMPLE = signs and symptoms, allergies, medications, pertinent medical history, injuries, illnesses, last meal/intake, events leading up to the injury and/or illness).

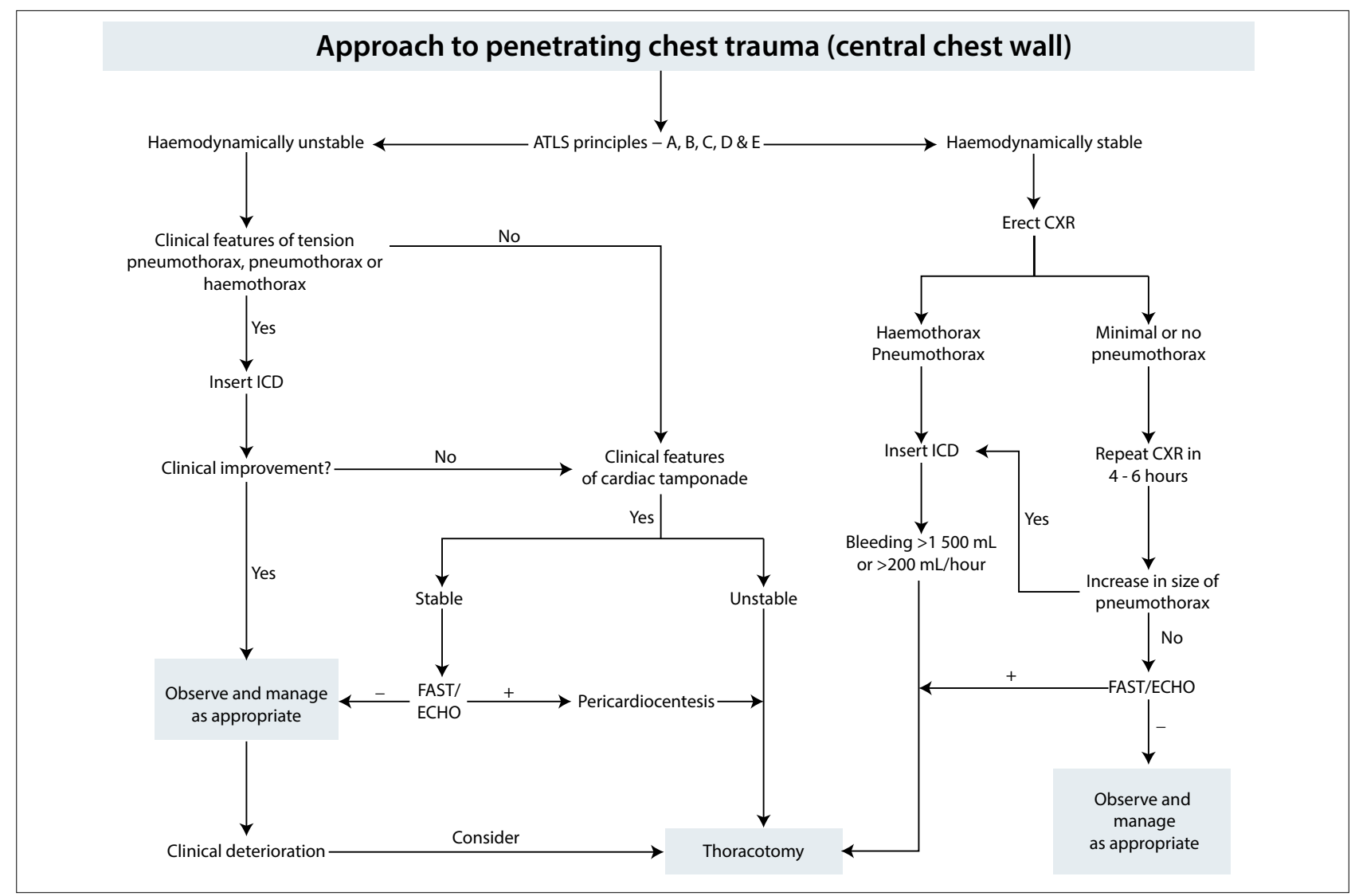

Fig. 3. Approach to penetrating chest trauma (central chest wall) (ATLS = advanced trauma life support; ICD = intercostal drain; FAST/ECHO = focused assessment with sonography in trauma/echocardiography; $C X R=$ chest $X$-ray). 


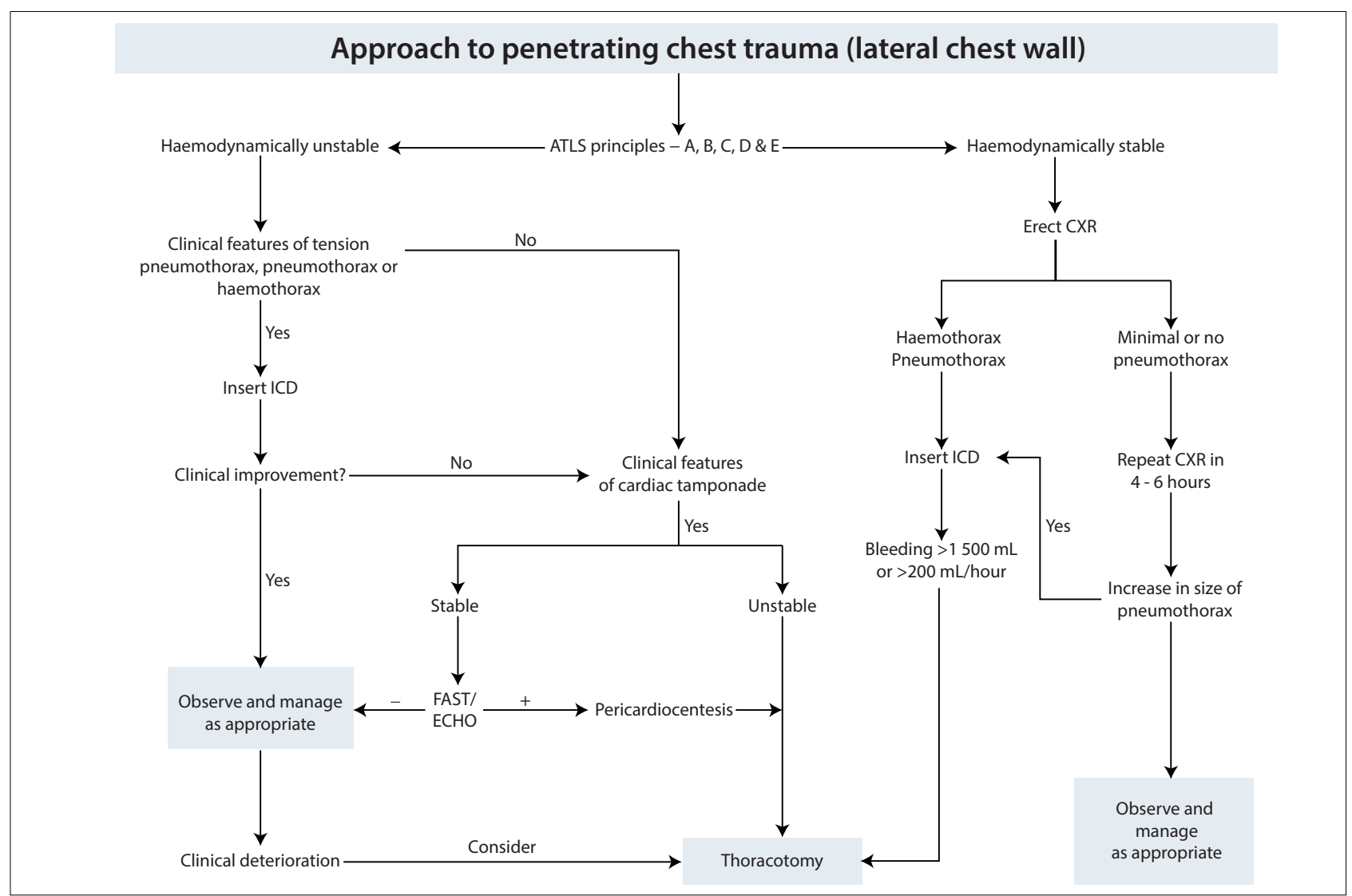

Fig. 4. Approach to penetrating chest trauma (lateral chest wall) $(A T L S=$ advanced trauma life support; ICD = intercostal drain; FAST/ECHO = focused assessment with sonography in trauma/echocardiography; $C X R=$ chest $X$-ray).

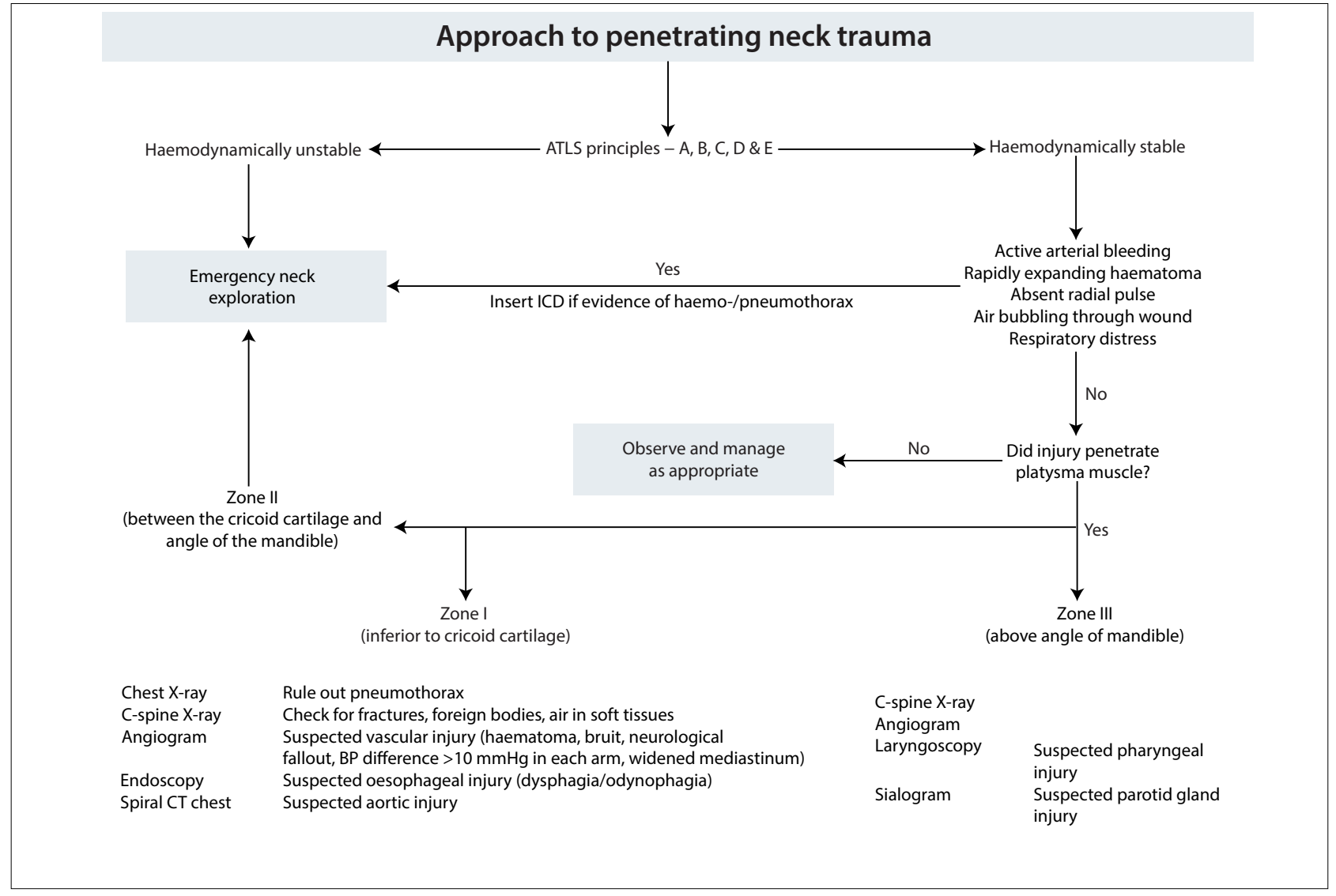

Fig. 5. Approach to penetrating neck trauma (ATLS = advanced trauma life support; ICD = intercostal drain; $C T=$ computed tomography; $C$-spine $=$ cervical spine; $B P=$ blood pressure). 


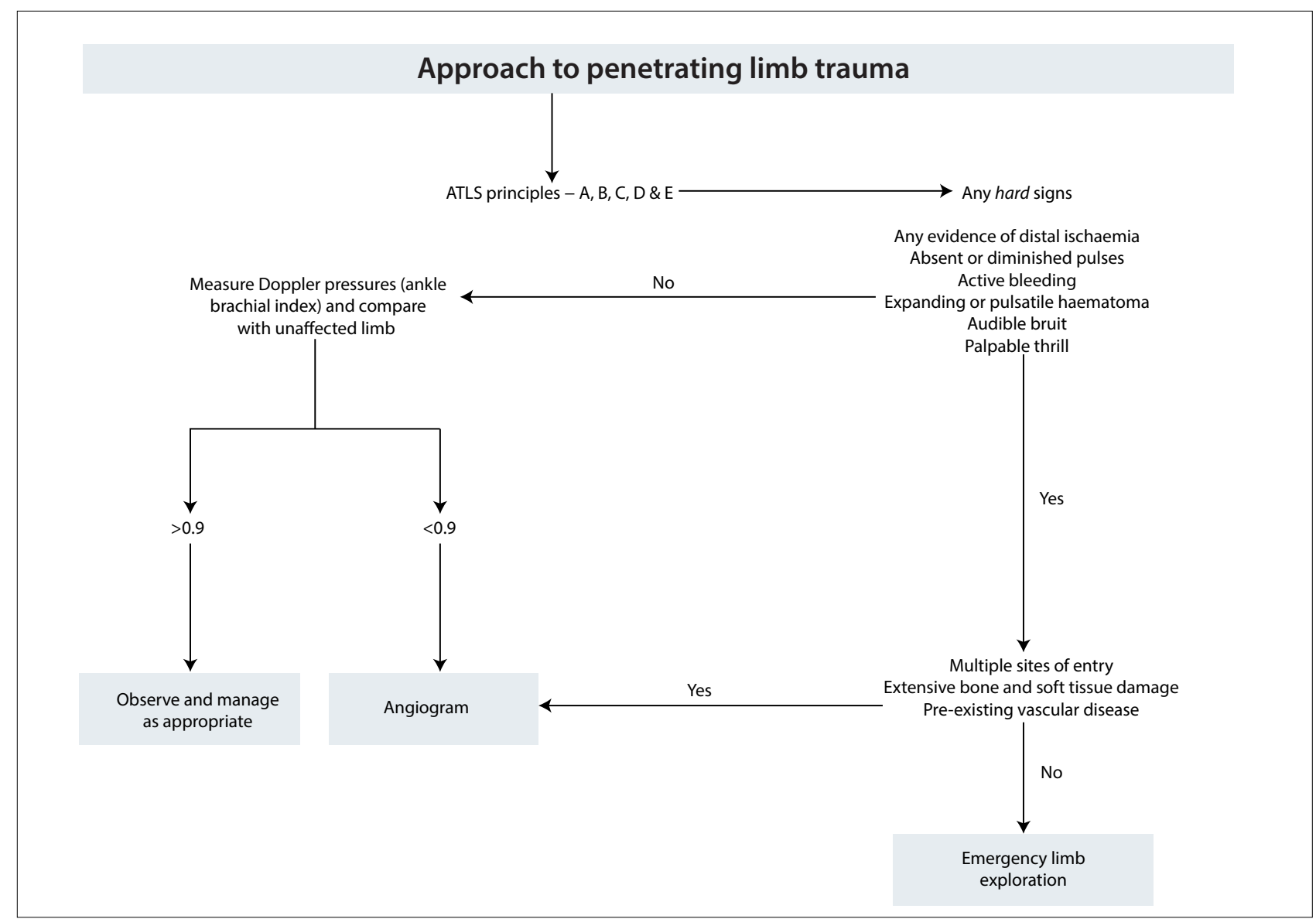

Fig. 6. Approach to penetrating limb trauma (ATLS = advanced trauma life support).

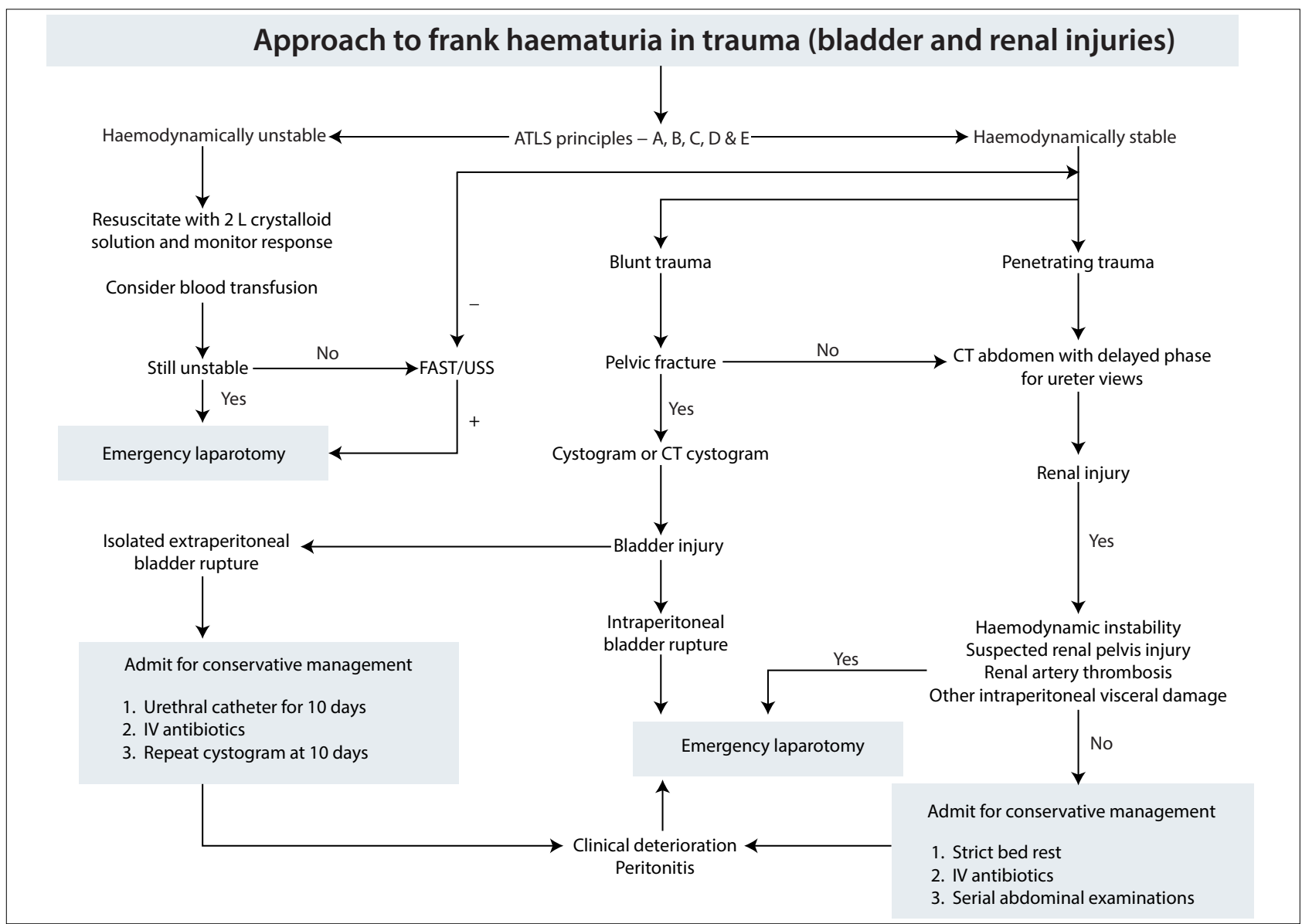

Fig. 7. Approach to frank haematuria in trauma (bladder and renal injuries) (ATLS = advanced trauma life support; IV = intravenous; FAST/USS = focused assessment with sonography in trauma/ultrasound scan; CT = computed tomography). 


\section{Approach to frank haematuria in trauma (urethral injuries)}

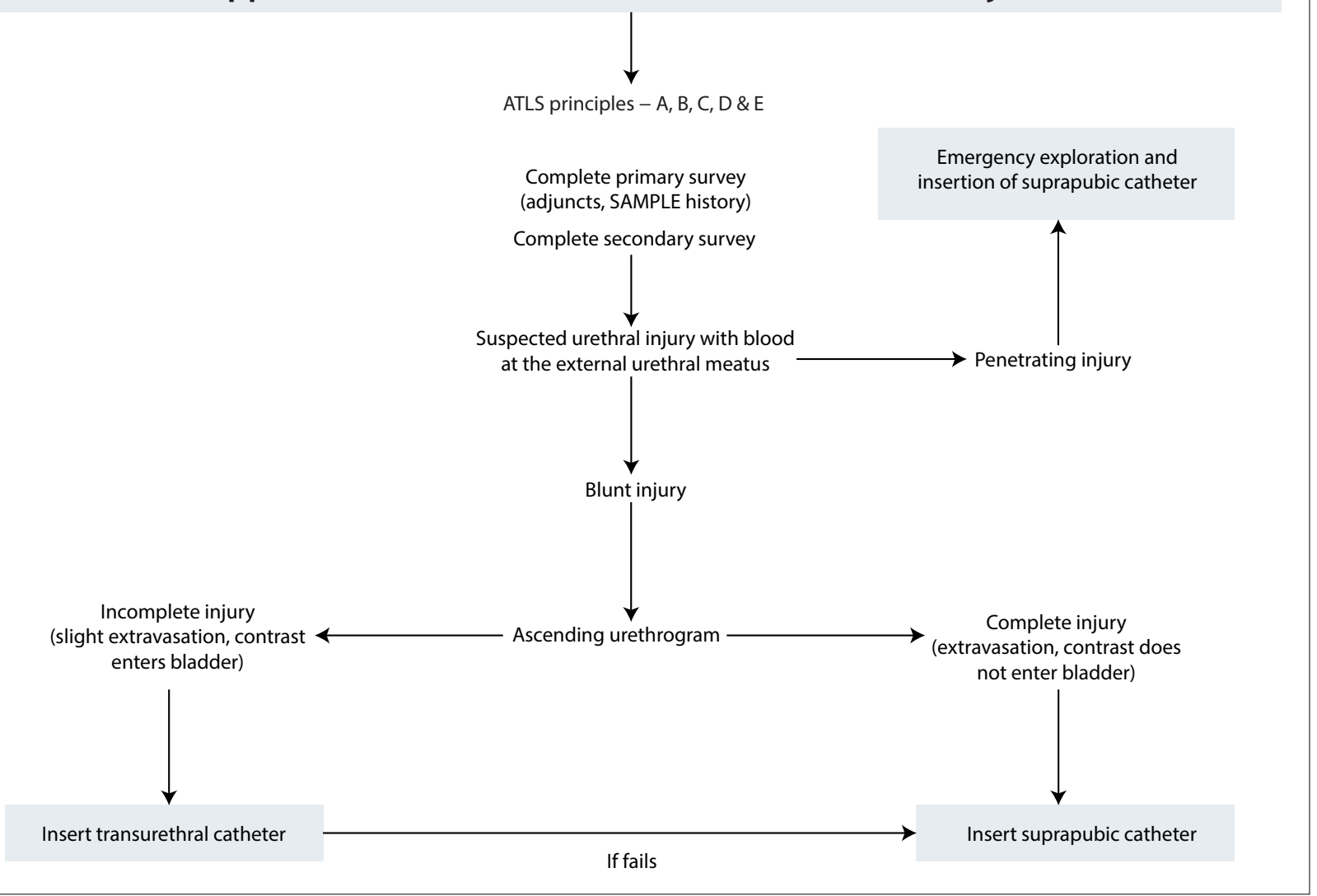

Fig. 8. Approach to frank haematuria in trauma (urethral injuries) (ATLS = advanced trauma life support; SAMPLE = signs and symptoms, allergies, medications, pertinent medical history, injuries, illnesses, last meal/intake, events leading up to the injury and/or illness).

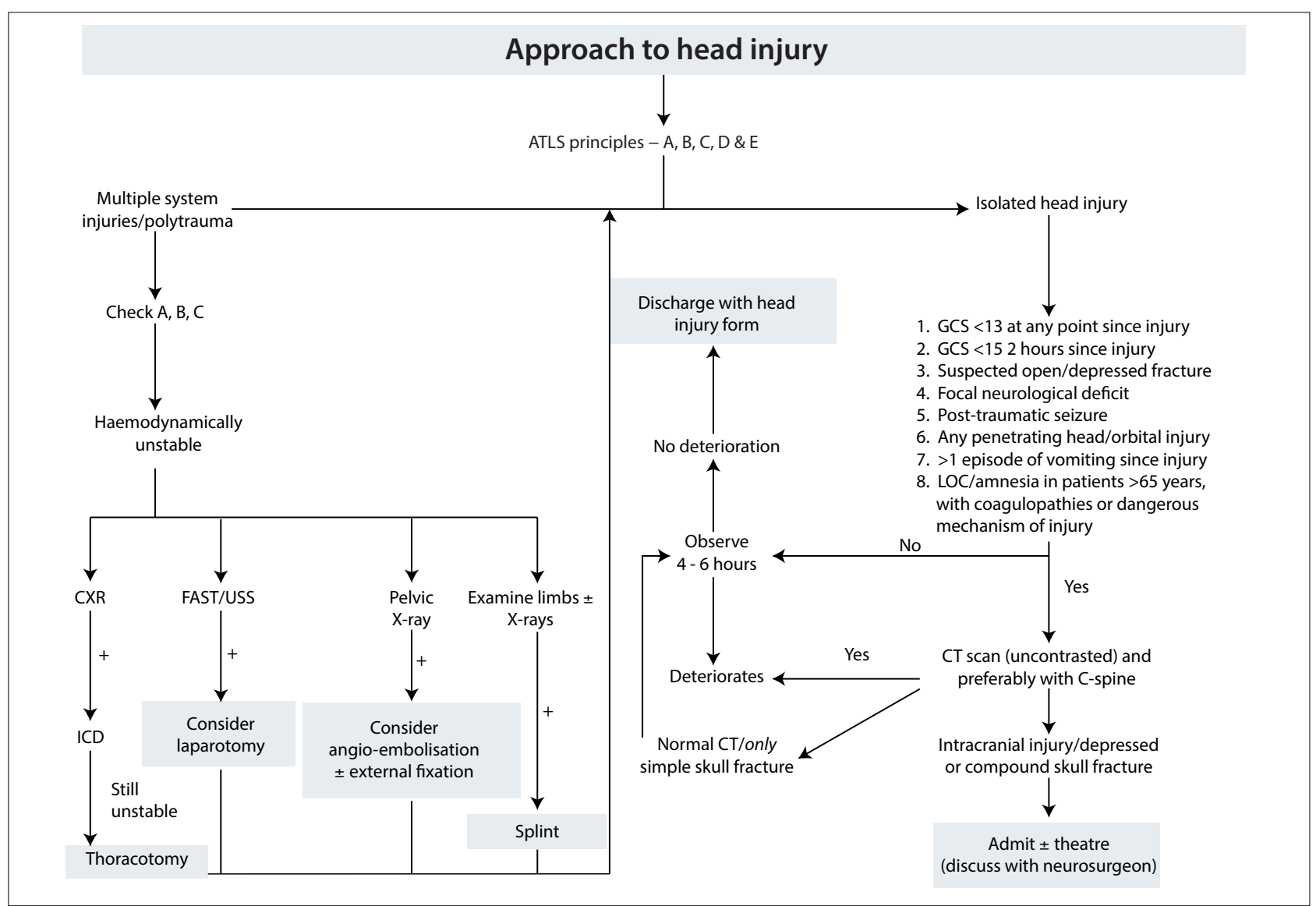

Fig. 9. Approach to head injury (ATLS = advanced trauma life support; CXR = chest X-ray; FAST/USS = focused assessment with sonography in trauma/ultrasound scan; ICD = intercostal drain; GCS = Glasgow Coma Scale; LOC = loss of consciousness; $C T=$ computed tomography; C-spine = cervical spine). 
Acknowledgement. A special thank you to the consultants at Frere Hospital for reviewing the algorithms and perfecting them for publication, especially Drs W Matshoba, K Kesner, E Simpson, D Brown and A Makangee. The contribution of the Eastern Cape Department of Health in providing funding for the printing and circulation of the algorithms and the Surgery Survival Guide handbook is gratefully acknowledged. A special word of appreciation to Prof. G Boon for driving this process from start to finish.

\section{Further reading}

American College of Surgeons, Committee on Trauma. ATLS: Advanced Trauma Life Support Program for Doctors (ATLS). Student Manual. 6th ed. Chicago, IL: American College of Surgeons, 1997. Heyns C, Barnes D. Introduction to Urology. Cape Town: South African Urology Association. John J, ed. Surgery Survival Guide. East London: Eastern Cape Department of Health, 2015. Nicol A, Steyn E, eds. Handbook of Trauma for Southern Africa. 3rd ed. Cape Town: Oxford University Press Southern Africa, 2010 .

Welzel T. Emergency Medicine Guidance for the Western Cape. Revised ed. Cape Town: Western Cape Department of Health, 2013

S Afr Med J 2015;105(6):502-507. DOI:10.7196/SAMJ.9795 